\title{
A RADON-NIKODÝM THEOREM FOR VECTOR POLYMEASURES
}

\author{
F. J. FERNÁNDEZ, P. JIMÉNEZ GUERRA and M. T. ULECIA
}

(Received 30 April 1998; revised 5 October 1998)

Communicated by P. G. Dodds

\begin{abstract}
A Radon-Nikodým theorem for Banach valued polymeasures is proved.

1991 Mathematics subject classification (Amer. Math. Soc.): primary 28B05, 46G10.
\end{abstract}

\section{Introduction}

A Radon-Nikodým theorem for (scalar) Radon polymeasures was given in [5]. The aim of this paper is to give a Radon-Nikodým theorem for (general) Banach valued polymeasures. Despite the fact that the Radon-Nikodým theorem proved here extends that of [9], Example 6 points out that a Radon-Nikodým theorem for polymeasures (inclusive, for scalar bimeasures) cannot be stated using a condition of localization which is just an extension of the Maynard's condition of localization in compacts. The integrable $d$-tuples used here belong to the first integrable class of [3], and their integrals coincide with that of [3].

Finally, let us point out that, as predicted in [7], polymeasures that do not determine a measure in the product space are increasingly appearing in areas as diverse as nonstationary processes, harmonic analysis, operator theory and quantum physics (see $[6])$.

\section{Notation and preliminaries}

In the following, $d$ will be a fixed positive integer, $\Sigma_{i}, i=1, \ldots, d$, will be $\sigma$-algebras of subsets of non empty sets $\Omega_{i}, X_{i}, i=1, \ldots, d, Y$ and $Z$ will be (C) 1999 Australian Mathematical Society 0263-6115/99 $\$ A 2.00+0.00$ 
given Banach spaces over the same scalar field $K$ of real or complex numbers, and $\phi: X_{1} \times \cdots \times X_{d} \times Y \rightarrow Z$ will be a continuous multilinear mapping, that without loss of generality, can be supposed to be such that $\|\phi\| \leq 1$. We also consider two fixed polymeasures (in the usual sense of [2]) $\alpha: \Sigma_{1} \times \cdots \times \Sigma_{d} \rightarrow Y$ and $\gamma: \Sigma_{1} \times \cdots \times \Sigma_{d} \rightarrow Z$.

Denote by $S_{i}\left(\Omega_{i}\right), i=1, \ldots, d$, the normed linear space of $\Sigma_{i}$-simple functions $f_{i}: \Omega_{i} \rightarrow X_{i}$ with the sup norm $\left\|f_{i}\right\|_{\Omega_{i}}=\sup \left\{\left|f_{i}\left(t_{i}\right)\right|: t_{i} \in \Omega_{i}\right\}$. Let $f_{i} \in S_{i}\left(\Omega_{i}\right), i=$ $1, \ldots, d$, be of the form $f_{i}=\sum_{j_{i}=1}^{r_{i}} x_{i, j_{i}} \chi_{A_{i}, j_{i}}$ with $x_{i, j_{i}} \in X_{i}$, and with pairwise disjoint $A_{i, j_{i}} \in \Sigma_{i}$. Then the integral of the $d$-tuple $\left(f_{1}, \ldots, f_{d}\right) \in S_{1}\left(\Omega_{1}\right) \times \cdots \times S_{d}\left(\Omega_{d}\right)$ over $\left(A_{1}, A_{2}, \ldots, A_{d}\right) \in \Sigma_{1} \times \cdots \times \Sigma_{d}$ is defined (see [2]) by

$$
\int_{\left(A_{1}, A_{2} \ldots, A_{d}\right)}\left(f_{1}, \ldots, f_{d}\right) d \alpha=\sum_{j_{1}=1}^{r_{1}} \ldots \sum_{j_{d}=1}^{r_{d}} \phi\left(x_{1, j_{1}}, \ldots, x_{d \cdot j_{d}}, \alpha\left(A_{1, j_{1}}, \ldots, A_{d, j_{d}}\right)\right) .
$$

In the sequel, if there is no confusion, we will simply write $S_{i},\left\|f_{i}\right\|, \int_{\left(A_{i}\right)}\left(f_{i}\right) d \alpha$, $\left(f_{i}\right) \in \times S_{i}$ and $\left(A_{i}\right) \in \times \Sigma_{i}$.

Following [2], we define the semivariation $\hat{\alpha}: \Sigma_{1} \times \cdots \times \Sigma_{d} \rightarrow[0,+\infty]$ and the variation $|\alpha|: \Sigma_{1} \times \cdots \times \Sigma_{d} \rightarrow[0,+\infty]$, of the polymeasure $\alpha$, by

$$
\hat{\alpha}\left(A_{1}, \ldots, A_{d}\right)=\sup \left\{\left\|\int_{\left(A_{i}\right)}\left(f_{i}\right) d \alpha\right\|: f_{i} \in S_{i},\left\|f_{i}\right\| \leq 1, i=1, \ldots, d\right\}
$$

and

$$
\begin{aligned}
&|\alpha|\left(A_{1}, \ldots, A_{d}\right)=\sup \{\left\{\sum_{j_{1}=1}^{r_{1}} \ldots \sum_{j_{d}=1}^{r_{d}}\left\|\alpha\left(A_{1, j_{1}}, \ldots, A_{d, j_{d}}\right)\right\|,\right. \\
&\left.A_{i, j_{i}} \in \Sigma_{i} \text { pairwise disjoint with } A_{i}=\bigcup_{j_{i}=1}^{r_{i}} A_{i, j_{i}}\right\} .
\end{aligned}
$$

Let us also consider the set function $\|\hat{\alpha}\|: \times \Sigma_{i} \rightarrow[0,+\infty]$ defined by

$$
\|\hat{\alpha}\|\left(A_{1}, \ldots, A_{d}\right)=\sum_{i=1}^{d}|\hat{\alpha}|\left(\Omega_{1}, \ldots, A_{i}, \ldots, \Omega_{d}\right) .
$$

In the following, let us assume that $\hat{\alpha}\left(\Omega_{1}, \ldots, \Omega_{d}\right)<+\infty$.

Definition 1. A function $f_{i}: \Omega_{i} \rightarrow X_{i}$ is said to be $u$-simple if it is a uniform limit of a sequence of simple functions from $\Omega_{i}$ into $X_{i}$. Denote by $U_{i}, i=1, \ldots, d$, the space of the $u$-simple functions $f_{i}: \Omega_{i} \rightarrow X_{i}$. 
If $\left(f_{i}\right) \in \times U_{i}$, then the integral of the $d$-tuple $\left(f_{1}, \ldots, f_{d}\right) \in U_{1} \times \cdots \times U_{d}$ over $\left(A_{i}\right) \in X \Sigma_{i}$ is defined by

$$
\int_{\left(A_{1}, A_{2}, \ldots, A_{d}\right)}\left(f_{1}, \ldots, f_{d}\right) d \alpha=\lim _{n \rightarrow \infty} \int_{\left(A_{1}, A_{2}, \ldots, A_{d}\right)}\left(f_{1}^{n}, \ldots, f_{d}^{n}\right) d \alpha,
$$

where $\left(f_{i}^{n}\right)_{n \in \mathbb{N}}, i=1, \ldots, d$, is any sequence in $S_{i}$ which is uniformly convergent to $f_{i}$. It is easily proved that this integral is well defined and it has all properties of the integral of $d$-tuples of simple functions.

A $d$-tuple of functions $f_{i}: \Omega_{i} \rightarrow X_{i}, i=1, \ldots, d$, is said to be $\alpha$-measurable, if for every $\varepsilon>0$ there exists $\left(A_{i}\right) \in \times \Sigma_{i}$ such that $\|\alpha\|\left(\Omega_{1}-A_{1}, \ldots, \Omega_{d}-A_{d}\right)<\varepsilon$ and $\left(f_{i} \chi_{A_{i}}\right) \in X U_{i}$.

An $\alpha$-measurable $d$-tuple $\left(f_{i}\right)$ is said to be $\alpha$-integrable, if for every $\varepsilon>0$ there exists $\delta>0$ such that for every $d$-tuple $\left(A_{i}\right) \in \times \Sigma_{i}$ satisfying $\hat{\alpha}\left(A_{1}, \ldots, A_{d}\right)<\delta$ and $\left(f_{i} \chi_{A_{i}}\right) \in \times U_{i}$, we have that

$$
\left\|\int_{\left(A_{i}\right)}\left(f_{i}\right) d \alpha\right\|<\varepsilon
$$

If the $d$-tuple $\left(f_{i}\right)$ is $\alpha$-integrable, then the integral of $\left(f_{i}\right)$ over $\left(A_{i}\right) \in \times \Sigma_{i}$ is defined by

$$
\int_{\left(A_{1}, A_{2}, \ldots, A_{d}\right)}\left(f_{1}, \ldots, f_{d}\right) d \alpha=\lim _{\substack{\left(B_{i}\right) \in \times \Sigma_{i} \cap n_{\varphi\left(A_{i}\right)} \\ U_{i} \times A_{i} \in \in \times v_{i}}} \int_{\left(B_{i}\right)}\left(f_{i}\right) d \alpha
$$

where $\wp\left(A_{i}\right)$ denotes the family of all subsets of $A_{i}(i=1, \ldots, d)$.

It is easily proved that the above integral is well defined. Furthermore, every $\alpha$-integrable $d$-tuple belongs to the first integrable class, defined in [3, pp. 592, 593], and its integrals in both senses coincide. Let us also point out, that this integral coincides, when $d=1$, with the vector integration introduced in [4].

\section{A Radon-Nikodým theorem}

Suppose that $0<|\alpha|\left(\Omega_{1}, \ldots, \Omega_{d}\right)<+\infty$.

Definition 2. Let $\left(A_{1}, \ldots, A_{d}\right) \in\left(\Sigma_{1} \times \cdots \times \Sigma_{d}\right)^{+}=\left\{\left(B_{i}\right) \in \times \Sigma_{i}:|\alpha|\left(B_{1}\right.\right.$, $\left.\left.\ldots, B_{d}\right)>0\right\}$. A mapping $\varphi:(0,+\infty) \rightarrow \times S_{i}\left(A_{i}\right)$ will be a localization for $\left(A_{1}, \ldots, A_{d}\right)$ if the following assertions hold:

(2.1) $\left\|\gamma\left(B_{1}, \ldots, B_{d}\right)-\int_{\left(B_{i}\right)} \varphi(\varepsilon) d \alpha\right\| \leq \varepsilon$, for every $\varepsilon \in(0,+\infty)$ and every $d$-tuple $\left(B_{i}\right) \in \times \Sigma_{i}$ such that $\left(B_{i}\right) \subseteq\left(A_{i}\right)$ (that is, $\left.B_{i} \subseteq A_{i}, i=1, \ldots, d\right)$. 
(2.2) $\left\|\pi_{i} \varphi(\varepsilon)-\pi_{i} \varphi\left(\varepsilon^{\prime}\right)\right\|_{A_{i}} \leq \varepsilon$, for every $0<\varepsilon^{\prime}<\varepsilon<+\infty$ and $i=1, \ldots, d$, where $\pi_{i}: \times S_{i}\left(A_{i}\right) \rightarrow S_{i}\left(A_{i}\right), i=1, \ldots, d$, denotes the usual projection $\left(\pi_{i}\left(f_{1}\right.\right.$, $\left.\left.\ldots, f_{d}\right)=f_{i}\right)$.

We say that a $d$-tuple $\left(A_{i}\right) \in\left(X \Sigma_{i}\right)^{+}$is localized, when there is a localization for it. A sequence of $d$-tuples $\left\{\left(A_{i}^{n}\right)\right\}_{n \in \mathbb{N}} \subseteq\left(X \Sigma_{i}\right)^{+}$is said to be localized if $\left(A_{1}^{n}, \ldots, A_{d}^{n}\right)$ is localized for every $n \in \mathbb{N}$.

If $\varphi_{j}$ is a localization for $\left(A_{i}^{j}\right) \in\left(X \Sigma_{i}\right)^{+}, j=1,2$, and $\left(A_{i}^{1}\right) \subseteq\left(A_{i}^{2}\right)$, we will say that $\varphi_{1}$ and $\varphi_{2}$ are consistent if $\left\|\pi_{i} \varphi_{1}(\varepsilon)-\pi_{i} \varphi_{2}(\varepsilon) \chi_{A_{i}^{i}}\right\|_{A_{i}^{\prime}} \leq \varepsilon, i=1, \ldots, d$, for every $\varepsilon>0$. Let $\left\{\left(A_{i}^{n}\right)\right\}_{n \in \mathbb{N}} \subseteq\left(X \Sigma_{i}\right)^{+}$be an increasing localized sequence of $d$-tuples. If there exists a localization $\varphi_{n}$ for $\left(A_{i}^{n}\right), n \in \mathbb{N}$, such that $\varphi_{n}$ and $\varphi_{m}$ are consistent for all $n, m \in \mathbb{N}$ with $m>n$, then we say that $\left\{\left(A_{i}^{n}\right)\right\}_{n \in \mathbb{N}}$ is consistent.

REMARK 3. A $d$-tuple $\left(A_{i}\right) \in\left(X \Sigma_{i}\right)^{+}$is localized if and only if there exist $d$ non empty compact subsets $K_{i} \subseteq X_{i}, i=1, \ldots, d$, such that for every $\varepsilon>0$ and every finite family $\left\{K_{i}^{j}\right\}_{j \in J_{i}}$ of non empty compact subsets of $X_{i}$ with diameter less than or equal $\varepsilon$, such that $K_{i}=\bigcup_{j \in J_{i}} K_{i}^{j}, i=1, \ldots, d$, there exists $\lambda>0$ (depending on $\varepsilon$ and $\left\{K_{i}\right\}_{i=1}^{d}$ ) such that:

(3.1) For every $i=1, \ldots, d$, there exists a measurable and finite partition $\left\{E_{i}^{j}\right\}_{j \in J_{i}}$ $\subseteq \Sigma_{i}$ of $\Omega_{i}$ and $x_{i}^{j} \in K_{i}^{j}, j \in J_{i}$, such that if $f_{i}=\sum_{j \in J_{i}} x_{i}^{j} \chi_{E_{i}^{j}}(i=1, \ldots, d)$, then

$$
\left\|\gamma\left(B_{1}, \ldots, B_{d}\right)-\int_{\left(B_{i}\right)}\left(f_{i}\right) d \alpha\right\| \leq \varepsilon \lambda|\alpha|\left(B_{1}, \ldots, B_{d}\right),
$$

for every $d$-tuple $\left(B_{i}\right) \in \times \Sigma_{i}$ with $\left(B_{i}\right) \subseteq\left(A_{i}\right)$.

(3.2) If (i) $0<\varepsilon^{\prime}<\varepsilon, i=1, \ldots, d$, and $\left\{C_{i}^{j}\right\}_{j \in J_{i}^{\prime}}$ is a finite family of non empty compact subsets of $X_{i}$, with diameter less than or equal $\varepsilon^{\prime}$, such that $K_{i}=\bigcup_{j \in J_{i}^{\prime}} C_{i}^{j}$, and (ii) for every $i=1, \ldots, d$ and every $j \in J_{i}^{\prime}$, there exists $h_{j} \in J_{i}$, with $C_{i}^{j} \subseteq K_{i}^{h_{j}}$, then there exists a finite partition $\left\{F_{i}^{j}\right\}_{j \in J_{i}^{\prime}} \subseteq \Sigma_{i}$ of $\Omega_{i}$ and $y_{i}^{j} \in C_{i}^{j}\left(j \in J_{i}^{\prime}\right)$ such that if $g_{i}=\sum_{j \in J_{i}^{\prime}} y_{i}^{j} \chi_{F_{i}^{j}}(i=1, \ldots, d)$, then $\left\|f_{i}-g_{i}\right\| \leq 2 \varepsilon$ for every $i=1, \ldots, d$ and $\left\|\gamma\left(B_{1}, \ldots, B_{d}\right)-\int_{\left(B_{i}\right)}\left(g_{i}\right) d \alpha\right\| \leq \varepsilon^{\prime} \lambda|\alpha|\left(B_{1}, \ldots, B_{d}\right)$, for every $\left(B_{i}\right) \in \times \Sigma_{i}$ with $\left(B_{i}\right) \subseteq\left(A_{i}\right)$.

THEOREM 4. The polymeasure $\gamma$ has a Radon-Nikodým derivative with respect to $\alpha$ (that is, there exists an $\alpha$-integrable d-tuple of functions $\left(f_{i}\right)$ such that $\gamma\left(A_{1}, \ldots\right.$, $\left.A_{d}\right)=\int_{\left(A_{i}\right)}\left(f_{i}\right) d \alpha$, for every $\left.\left(A_{i}\right) \in \times \Sigma_{i}\right)$ if and only if the following assertions hold:

(4.1) $\|\gamma\| \ll \hat{\alpha}$ (that is, $\lim \left\|\gamma\left(A_{1}, \ldots, A_{d}\right)\right\|=0$ as $\hat{\alpha}\left(A_{1}, \ldots, A_{d}\right) \rightarrow 0$, $\left.\left(A_{i}\right) \in \times \Sigma_{i}\right)$.

(4.2) There exists a consistent sequence $\left\{\left(A_{i}^{n}\right)\right\}_{n \in \mathbb{N}} \subseteq\left(X \Sigma_{i}\right)^{+}$such that $\|\alpha\|\left(\Omega_{1}-\right.$ $\left.\bigcup_{n \in \mathbb{N}} A_{1}^{n}, \ldots, \Omega_{d}-\bigcup_{n \in \mathbb{N}} A_{d}^{n}\right)=0$. 
Proof. Suppose that there exists a Radon-Nikodým derivative $\left(f_{1}, \ldots, f_{d}\right)$ of $\gamma$ with respect to $\alpha$. Since (4.1) follows immediately from the properties of the integral, let us prove (4.2). In fact, since $\left(f_{1}, \ldots, f_{d}\right)$ is $\alpha$-measurable, there exists $\left(B_{i}^{n}\right) \in \times \Sigma_{i}, n \in \mathbb{N}$, such that

$$
\|\alpha\|\left(\Omega_{1}-B_{1}^{n}, \ldots, \Omega_{d}-B_{d}^{n}\right)<\frac{|\alpha|\left(\Omega_{1}, \ldots, \Omega_{d}\right)}{1+n}
$$

and $\left(f_{i} \chi_{B_{i}^{n}}\right) \in \times U_{i}$.

If $A_{i}^{n}=\bigcup_{1 \leq m \leq n} B_{i}^{m}$ for every $n \in \mathbb{N}$ and every $i=1, \ldots, d$, then

$$
|\alpha|\left(A_{1}^{n}, \ldots, A_{d}^{n}\right) \geq|\alpha|\left(B_{1}^{1}, \ldots, B_{d}^{1}\right)>\frac{1}{2}|\alpha|\left(\Omega_{1}, \ldots, \Omega_{d}\right)>0
$$

and $\left\{\left(A_{i}^{n}\right)\right\}_{n \in \mathbb{N}} \subseteq\left(\times \Sigma_{i}\right)^{+}$.

Furthermore,

$$
\begin{aligned}
\|\alpha\|\left(\Omega_{1}-\bigcup_{n \in \mathbb{N}} A_{1}^{n}, \ldots, \Omega_{d}-\bigcup_{n \in \mathbb{N}} A_{d}^{n}\right) & \leq\|\alpha\|\left(\Omega_{1}-B_{1}^{m}, \ldots, \Omega_{d}-B_{d}^{m}\right) \\
& \leq \frac{|\alpha|\left(\Omega_{1}, \ldots, \Omega_{d}\right)}{1+m}
\end{aligned}
$$

for every $m \in \mathbb{N}$, and therefore,

$$
\|\alpha\|\left(\Omega_{1}-\bigcup_{n \in \mathbb{N}} A_{1}^{n}, \ldots, \Omega_{d}-\bigcup_{n \in \mathbb{N}} A_{d}^{n}\right)=0
$$

Thus we have only to prove that the increasing sequence $\left\{\left(A_{i}^{n}\right)\right\}_{n \in \mathbb{N}}$ is consistent. Let us prove first that $\left(A_{1}^{n}, \ldots, A_{d}^{n}\right)$ is localized for every $n \in \mathbb{N}$. In fact, since $\left(f_{i} \chi_{A_{i}^{n}}\right) \in X U_{i}$ (and therefore, $\left\|f_{i}\right\|_{A_{i}^{n}}<+\infty$, for $\left.i=1, \ldots, d\right)$, for every $\varepsilon>0$ there exists $\left(g_{i}^{n, \varepsilon}\right) \in \times S_{i}\left(A_{i}^{n}\right)$ such that

$$
\left\|f_{i} \chi_{A_{i}^{n}}-g_{i}^{n \cdot \varepsilon}\right\|_{A_{i}^{n}} \leq \min \left[1, \varepsilon /\left[2 d\left(1+\max _{1 \leq i \leq d}\left\|f_{i}\right\|_{A_{i}^{n}}\right)^{d}\left(1+\hat{\alpha}\left(A_{1}, \ldots, A_{d}\right)\right)\right]\right]
$$

for every $i=1, \ldots, d$. Let $\varphi_{n}:(0,+\infty) \rightarrow \times S_{i}\left(A_{i}^{n}\right)$ be the function defined by $\varphi_{n}(\varepsilon)=\left(g_{1}^{n, \varepsilon}, \ldots, g_{d}^{n, \varepsilon}\right)$ for every $\varepsilon>0$.

If $\left(B_{i}\right) \in \times \Sigma_{i}$ verify that $\left(B_{i}\right) \subseteq\left(A_{i}^{n}\right)$, then

$$
\begin{aligned}
& \left\|\gamma\left(B_{1}, \ldots, B_{d}\right)-\int_{\left(B_{i}\right)} \varphi_{n}(\varepsilon) d \alpha\right\| \\
& =\left\|\int_{\left(B_{i}\right)}\left(f_{i}\right) d \alpha-\int_{\left(B_{i}\right)}\left(g_{i}^{n, \varepsilon}\right) d \alpha\right\|
\end{aligned}
$$




$$
\begin{aligned}
& \leq\left\|\int_{\left(B_{i}\right)}\left(f_{1-} g_{1}^{n, \varepsilon}, f_{2}, \ldots, f_{d}\right) d \alpha\right\| \\
& +\cdots+\left\|\int_{\left(B_{i}\right)}\left(g_{1}^{n, \varepsilon}, \ldots, g_{d-1}^{n, \varepsilon}, f_{d}-g_{d}^{n, \varepsilon}\right) d \alpha\right\| \\
& \leq \frac{d\left(\prod_{i=1}^{d}\left(1+\left\|f_{i}\right\|_{A_{i}^{n}}\right)\right) \varepsilon \hat{\alpha}\left(B_{1}, \ldots, B_{d}\right)}{2 d\left(1+\max _{1 \leq i \leq d}\left\|f_{i}\right\|_{A_{i}^{n}}\right)^{d}\left(1+\hat{\alpha}\left(A_{1}, \ldots, A_{d}\right)\right)} \\
& <\varepsilon \text {. }
\end{aligned}
$$

Furthermore, for every $0<\varepsilon^{\prime}<\varepsilon, n, m \in \mathbb{N}$ with $n<m$ and $i=1, \ldots, d$, we have

$$
\left\|g_{i}^{n, \varepsilon}-g_{i}^{n, \varepsilon^{\prime}}\right\|_{A_{i}^{n}} \leq\left\|g_{i}^{n, \varepsilon}-f_{i}\right\|_{A_{i}^{n}}+\left\|f_{i}-g_{i}^{n, \varepsilon^{\prime}}\right\|_{A_{i}^{n}}<\varepsilon
$$

and

$$
\left\|g_{i}^{m, \varepsilon}-g_{i}^{n, \varepsilon}\right\|_{A_{i}^{n}} \leq\left\|g_{i}^{m, \varepsilon}-f_{i}\right\|_{A_{i}^{m}}+\left\|f_{i}-g_{i}^{n, \varepsilon}\right\|_{A_{i}^{n}} \leq \varepsilon .
$$

Therefore (4.2) holds.

Conversely, assume that (4.1) and (4.2) are verified. Let $\left\{\left(A_{i}^{n}\right)\right\}_{n \in \mathbb{N}} \subseteq\left(X \Sigma_{i}\right)^{+}$be a consistent sequence verifying that

$$
\|\alpha\|\left(\Omega_{1}-\bigcup_{n \in \mathbb{N}} A_{1}^{n}, \ldots, \Omega_{d}-\bigcup_{n \in \mathbb{N}} A_{d}^{n}\right)=0 .
$$

For every $n \in \mathbb{N}$, let $\varphi_{n}:(0,+\infty) \rightarrow \times S_{i}\left(A_{i}^{n}\right)$ be a localization for $\left(A_{i}^{n}\right)$ such that $\varphi_{n}$ and $\varphi_{m}$ are consistent for all $m, n \in \mathbb{N}$ with $n<m$. Let us set $\varphi_{n}(1 / m)=$ $\left(g_{1}^{n, m}, \ldots, g_{d}^{n, m}\right) \in X S_{i}\left(A_{i}^{n}\right)$. Since $\left\|g_{i}^{n, m}(t)-g_{i}^{n, p}(t)\right\| \leq 1 / m$ for every $m, n, p \in \mathbb{N}$ with $m \leq p$ and every $t \in A_{i}^{n}$, it follows that $\left(g_{i}^{n, m}(t)\right)_{m \in \mathbb{N}}$ is a Cauchy sequence for every $t \in A_{i}^{n}$. Let

$$
g_{i}^{n}(t)=\lim _{m} g_{i}^{n, m}(t)
$$

for every $t \in A_{i}^{n}$. The above limit is uniform on $A_{i}^{n}$, and thus the function $g_{i}^{n}: A_{i}^{n} \rightarrow X_{i}$ is $u$-simple. Furthermore, $\left.g_{i}^{m}\right|_{A_{i}^{n}}=g_{i}^{n}$ for all $m, n \in \mathbb{N}$ with $m>n$, because

$$
\begin{aligned}
\left\|g_{i}^{n}(t)-g_{i}^{m}(t)\right\| & \leq\left\|g_{i}^{n}(t)-g_{i}^{n, p}(t)\right\|+\left\|g_{i}^{n, p}(t)-g_{i}^{m, p}(t)\right\|+\left\|g_{i}^{m, p}(t)-g_{i}^{m}(t)\right\| \\
& \leq 3 / p
\end{aligned}
$$

for every $p \in \mathbb{N}$. 
If $n \in \mathbb{N},\left(E_{i}\right) \in \times \Sigma_{i}$ and $\left(E_{i}\right) \subseteq\left(A_{i}\right)$, then

$$
\begin{aligned}
\left\|\gamma\left(E_{1}, \ldots, E_{d}\right)-\int_{\left(E_{i}\right)}\left(g_{i}^{n}\right) d \alpha\right\| & =\lim _{m}\left\|\gamma\left(E_{1}, \ldots, E_{d}\right)-\int_{\left(E_{i}\right)}\left(g_{i}^{n, m}\right) d \alpha\right\| \\
& \leq \lim _{m}\left(\frac{1}{m}\right)=0,
\end{aligned}
$$

and therefore,

$$
\gamma\left(E_{1}, \ldots, E_{d}\right)=\int_{\left(E_{i}\right)}\left(g_{i}^{n}\right) d \alpha
$$

For every $i=1, \ldots, d$, consider the function $f_{i}: \Omega_{i} \rightarrow X_{i}$ defined by

$$
f_{i}(t)= \begin{cases}g_{i}^{n}(t), & \text { if there exists } n \in \mathbb{N} \text { such that } t \in A_{i}^{n} \\ 0, & \text { if } t \in \Omega_{i}-\bigcup_{n \in \mathbb{N}} A_{i}^{n} .\end{cases}
$$

Let us prove that $\left(f_{1}, \ldots, f_{d}\right)$ is $\alpha$-measurable. In fact, since

$$
\|\alpha\|\left(\Omega_{1}-\bigcup_{n \in \mathbb{N}} A_{1}^{n}, \ldots, \Omega_{d}-\bigcup_{n \in \mathbb{N}} A_{d}^{n}\right)=0
$$

and $|\alpha|$ is a positive $d$-polymeasure, for every $\varepsilon>0$ there exist $r_{1}, \ldots, r_{d} \in \mathbb{N}$ verifying that

$$
|\alpha|\left(\Omega_{1}, \ldots, \Omega_{i}-A_{i}^{r_{i}}, \ldots, \Omega_{d}\right)=|\alpha|\left(\Omega_{1}, \ldots,\left(\bigcup_{n \in \mathbb{N}} A_{i}^{n}\right)-A_{i}^{r_{i}}, \ldots, \Omega_{d}\right)<\frac{\varepsilon}{d} .
$$

Thus setting $r=\max \left\{r_{1}, \ldots, r_{d}\right\}$ we have

$$
\|\alpha\|\left(\Omega_{1}-A_{1}^{r}, \ldots, \Omega_{d}-A_{d}^{r}\right)<\varepsilon
$$

and the function $\left.f_{i}\right|_{A_{i}^{r}}=g_{i}^{r}$ is $u$-simple, for $i=1, \ldots, d$.

Let us prove now that $\left(f_{1}, \ldots, f_{d}\right)$ is $\alpha$-integrable. Let $\varepsilon>0$. Since $\|\gamma\| \ll \hat{\alpha}$, there exists $\delta>0$ such that $\left\|\gamma\left(B_{1}, \ldots, B_{d}\right)\right\|<\varepsilon / 2$ for every $\left(B_{i}\right) \in \times \Sigma_{i}$ with $\hat{\alpha}\left(B_{1}, \ldots, B_{d}\right) \leq \delta$. Let $\left(E_{i}\right) \in\left(X \Sigma_{i}\right)$ be such that $\hat{\alpha}\left(E_{1}, \ldots, E_{d}\right) \leq \delta$ and $\left(f_{1} \chi_{A_{1}}, \ldots, f_{d} \chi_{A_{d}}\right) \in \times U_{i}$. Then, there exists $K>0$ such that $\left\|f_{i}\right\|_{E_{i}} \leq K$ for all $i=1, \ldots, d$. Proceeding as before, we can find $m \in \mathbb{N}$ such that

$$
\|\alpha\|\left(\Omega_{1}-A_{1}^{m}, \ldots, \Omega_{d}-A_{d}^{m}\right)<\frac{\varepsilon}{2 K^{d}} .
$$


Therefore,

$$
\begin{aligned}
& \left\|\int_{\left(E_{i}\right)}\left(f_{i}\right) d \alpha\right\| \\
& \leq\left\|\int_{\left(E_{1}-A_{1}^{m}, E_{2}, \ldots, E_{d}\right)}\left(f_{i}\right) d \alpha\right\|+\left\|\int_{\left(E_{1} \cap A_{1}^{m}, E_{2}-A_{2}^{m}, E_{3}, \ldots, E_{d}\right)}\left(f_{i}\right) d \alpha\right\| \\
& \quad+\cdots+\left\|\int_{\left(E_{1} \cap A_{1}^{m}, \ldots, E_{d-1} \cap A_{d-1}^{m}, E_{d}-A_{d}^{m}\right)}\left(f_{i}\right) d \alpha\right\|+\left\|\int_{\left(E_{1} \cap A_{1}^{m}, \ldots, E_{d} \cap A_{d}^{m}\right)}\left(f_{i}\right) d \alpha\right\| \\
& \leq \quad\left\|f_{1}\right\|_{E_{1}} \cdots\left\|f_{d}\right\|_{E_{d}}\left[\hat{\alpha}\left(E_{1}-A_{1}^{m}, E_{2}, \ldots, E_{d}\right)\right. \\
& \quad+\hat{\alpha}\left(E_{1} \cap A_{1}^{m}, E_{2}-A_{2}^{m}, E_{3}, \ldots, E_{d}\right) \\
& \left.\quad+\cdots+\hat{\alpha}\left(E_{1} \cap A_{1}^{m}, \ldots, E_{d-1} \cap A_{d-1}^{m}, E_{d}-A_{d}^{m}\right)\right] \\
& \quad+\left\|\int_{\left(E_{1} \cap A_{1}^{m} \ldots, E_{d} \cap A_{d}^{m}\right)}\left(g_{i}^{m}\right) d \alpha\right\| \\
& \leq
\end{aligned}
$$

and the $d$-tuple $\left(f_{1}, \ldots, f_{d}\right)$ is $\alpha$-integrable.

Let us see now that $\left(f_{1}, \ldots, f_{d}\right)$ is a Radon-Nikodým derivative of $\gamma$ with respect to $\alpha$. In fact, since $\|\alpha\| \|\left(\Omega_{1}-\bigcup_{n \in \mathbb{N}} A_{1}^{n}, \ldots, \Omega_{d}-\bigcup_{n \in \mathbb{N}} A_{d}^{n}\right)=0$, we have that

$$
\sum_{i=1}^{d} \hat{\alpha}\left(\Omega_{1}, \ldots, \Omega_{i}-\bigcup_{n \in \mathbb{N}} A_{i}^{n}, \ldots, \Omega_{d}\right)=0
$$

And having in mind that $\|\gamma\| \ll \hat{\alpha}$, we obtain that

$$
\|\gamma\|\left(\Omega_{1}-\bigcup_{n \in \mathbb{N}} A_{1}^{n}, \ldots, \Omega_{d}-\bigcup_{n \in \mathbb{N}} A_{d}^{n}\right)=0 .
$$

Therefore, for every $\left(E_{1}, \ldots, E_{d}\right) \in \times \Sigma_{i}$ and $m \in \mathbb{N}$ we have

$$
\begin{aligned}
& \left\|\gamma\left(E_{1}, \ldots, E_{d}\right)-\gamma\left(E_{1} \cap A_{1}^{m}, \ldots, E_{d} \cap A_{d}^{m}\right)\right\| \\
& \leq\|\| \gamma \|\left(\Omega_{1}-A_{1}^{m}, \ldots, \Omega_{d}-A_{d}^{m}\right)
\end{aligned}
$$




$$
\begin{aligned}
= & \|\gamma\|\left(\Omega_{1}-\bigcup_{n \in \mathbb{N}} A_{1}^{n}, \ldots, \Omega_{d}-\bigcup_{n \in \mathbb{N}} A_{d}^{n}\right) \\
& +\|\gamma\|\left(\bigcup_{n \in \mathbb{N}} A_{1}^{n}-A_{1}^{m}, \ldots, \bigcup_{n \in \mathbb{N}} A_{d}^{n}-A_{d}^{m}\right) \\
= & \|\gamma\|\left(\bigcup_{n \in \mathbb{N}} A_{1}^{n}-A_{1}^{m}, \ldots, \bigcup_{n \in \mathbb{N}} A_{d}^{n}-A_{d}^{m}\right) .
\end{aligned}
$$

Since the sequence $\left(\|\gamma\|\left(\bigcup_{n \in \mathbb{N}} A_{1}^{n}-A_{1}^{m}, \ldots, \bigcup_{n \in \mathbb{N}} A_{d}^{n}-A_{d}^{m}\right)\right)_{m \in \mathbb{N}}$ is convergent to zero (because the variation $|\gamma|$ of $\gamma$ is a positive polymeasure), it follows that

$$
\gamma\left(E_{1}, \ldots, E_{d}\right)=\lim _{m \rightarrow+\infty} \gamma\left(E_{1} \cap A_{1}^{m}, \ldots, E_{d} \cap A_{d}^{m}\right)
$$

for every $\left(E_{i}\right) \in \times \Sigma_{i}$.

Furthermore, for every $\varepsilon>0$ there exists $\delta>0$ such that

$$
\left\|\int_{\left(L_{1}, \ldots, L_{d}\right)}\left(f_{i}\right) d \alpha\right\|<\frac{\varepsilon}{d}
$$

for every $\left(L_{i}\right) \in \times \Sigma_{i}$ verifying that $\hat{\alpha}\left(L_{1}, \ldots, L_{d}\right) \leq \delta$. Then, noting that the sequence $\left(\sum_{i=1}^{d} \hat{\alpha}\left(\Omega_{1}, \ldots, \Omega_{i}-A_{i}^{m}, \ldots, \Omega_{d}\right)\right)_{m \in \mathbb{N}}$ converges to zero (because $\left(\|\alpha\|\left(\Omega_{1}-A_{1}^{m}, \ldots, \Omega_{d}-A_{d}^{m}\right)\right)_{m \in \mathbb{N}}$ is convergent to zero), it results the existence of $n_{0} \in \mathbb{N}$ such that

$$
\sum_{i=1}^{d} \hat{\alpha}\left(\Omega_{1}, \ldots, \Omega_{i}-A_{i}^{n}, \ldots, \Omega_{d}\right)<\delta
$$

for every $n \geq n_{0}$. Therefore, for every $\left(E_{i}\right) \in \times \Sigma_{i}$ we have

$$
\begin{aligned}
& \left\|\int_{\left(E_{1}, \ldots, E_{d}\right)}\left(f_{i}\right) d \alpha-\int_{\left(E_{1} \cap A_{1}^{n}, \ldots, E_{d} \cap A_{d}^{n}\right)}\left(f_{i}\right) d \alpha\right\| \\
& \leq\left\|\int_{\left(E_{1}-A_{1}^{n}, E_{2}, \ldots, E_{d}\right)}\left(f_{i}\right) d \alpha\right\|+\left\|\int_{\left(E_{1} \cap A_{1}^{n}, E_{2}-A_{2}^{n}, E_{3}, \ldots, E_{d}\right)}\left(f_{i}\right) d \alpha\right\| \\
& \quad+\cdots+\left\|\int_{\left(E_{1} \cap A_{1}^{n}, \ldots, E_{d-1} \cap A_{d-1}^{n}, E_{d}-A_{d}^{n}\right)}\left(f_{i}\right) d \alpha\right\| \\
& \quad \leq d \frac{\varepsilon}{d}=\varepsilon
\end{aligned}
$$

and consequently,

$$
\int_{\left(E_{i}\right)}\left(f_{i}\right) d \alpha=\lim _{n \rightarrow+\infty} \int_{\left(E_{1} \cap A_{1}^{n}, \ldots, E_{d} \cap A_{d}^{n}\right)}\left(f_{i}\right) d \alpha
$$


Now it follows from(4.5), (4.3) and (4.4) that

$$
\begin{aligned}
\int_{\left(E_{1}, \ldots, E_{d}\right)}\left(f_{i}\right) d \alpha & =\lim _{n \rightarrow+\infty} \int_{\left(E_{1} \cap A_{1}^{n}, \ldots, E_{d} \cap A_{d}^{n}\right)}\left(f_{i}\right) d \alpha \\
& =\lim _{n \rightarrow+\infty} \int_{\left(E_{1} \cap A_{1}^{n}, \ldots, E_{d} \cap A_{d}^{n}\right)}\left(g_{i}^{n}\right) d \alpha \\
& =\lim _{n \rightarrow+\infty} \gamma\left(E_{1} \cap A_{1}^{n}, \ldots, E_{d} \cap A_{d}^{n}\right) \\
& =\gamma\left(E_{1}, \ldots, E_{d}\right)
\end{aligned}
$$

for every $\left(E_{i}\right) \in X \Sigma_{i}$. Consequently, the $d$-tuple $\left(f_{i}\right)$ is a Radon-Nikodým derivative of the polymeasure $\gamma$ with respect to the polymeasure $\alpha$.

REMARK 5. In view of condition (iii) of [9, Theorem 2.1] and Remark 3, it is natural to consider the possibility of replacing condition (4.2) in Theorem 4 by the following:

(4.6) For every $\left(A_{i}\right) \in\left(X \Sigma_{i}\right)^{+}$, there exists a localized $d$-tuple $\left(B_{i}\right) \in\left(X \Sigma_{i}\right)^{+}$ such that $\left(B_{i}\right) \subseteq\left(A_{i}\right)$.

Conditions (4.2) and (4.6) are equivalent if $d=1$, but the following example shows that it is not possible, in general, to replace (4.2) by (4.6) in Theorem 4 when $d \geq 2$.

EXAMPLE 6. Let us consider $\Omega_{i}=[-1,1], \Sigma_{i}$ the Borel $\sigma$-algebra on $[-1,1]$, $i=1,2, X_{1}=X_{2}=Y=Z=\mathbb{R}, \phi: \mathbb{R} \times \mathbb{R} \times \mathbb{R} \rightarrow \mathbb{R}$ the ordinary product of scalars and $\alpha: \Sigma_{1} \times \Sigma_{2} \rightarrow \mathbb{R}$ the restriction to $\Sigma_{1} \times \Sigma_{2}$ of the Lebesgue measure $\alpha_{1}$ on $\operatorname{Bor}\left(\Omega_{1} \times \Omega_{2}\right)$.

Let $\gamma_{1}$ be the measure defined on the Borel $\sigma$-algebra of $\Omega_{1} \times \Omega_{2}$, by

$$
\gamma_{1}(B)=\int_{B} f d \alpha_{1}
$$

where $f=1-\chi_{[0,1] \times[0,1]}$.

If $\gamma$ denotes the restriction of $\gamma_{1}$ to $\Sigma_{1} \times \Sigma_{2}$, then it is easily proved that $\gamma$ is a bimeasure verifying (4.1) and (4.6).

Let us prove that $\gamma$ has no Radon-Nikodým derivative with respect to $\alpha$. In fact, if there exists an $\alpha$-integrable 2 -tuple $\left(f_{1}, f_{2}\right)$ such that

$$
\gamma\left(A_{1}, A_{2}\right)=\int_{\left(A_{1}, A_{2}\right)}\left(f_{1}, f_{2}\right) d \alpha
$$

for every $\left(A_{1}, A_{2}\right) \in \Sigma_{1} \times \Sigma_{2}$, then $f_{1} f_{2}$ is $\alpha_{1}$-integrable and

$$
\int_{A \times B} f d \alpha_{1}=\int_{A \times B}\left(f_{1} f_{2}\right) d \alpha_{1}
$$


for every $(A, B) \in \Sigma_{1} \times \Sigma_{2}$.

It follows now from the Fubini theorem that there exists $D \in \Sigma_{1}$ such that $\lambda(D)=0$, where $\lambda$ denotes the Lebesgue measure on $[-1,1], f_{1}(x) f_{2}$ is $\alpha$-integrable for every $x \in \Omega_{1} \backslash D$ and

$$
\int_{B}\left(f(x, \cdot)-f_{1}(x) f_{2}\right) d y=0
$$

for every $B \in \Sigma_{2}$ and every $x \in \Omega_{1} \backslash D$. Therefore, there exists $E \in \Sigma_{2}$ such that $\lambda(E)=0$ and $f(x, y)=f_{1}(x) f_{2}(y)$ for every $(x, y) \in\left(\Omega_{1} \times \Omega_{2}\right) \backslash(D \times E)$.

Since $\lambda(D)=\lambda(E)=0$, there exists $x_{1} \in[0,1] \backslash D, y_{1} \in[0,1] \backslash E, x_{2} \in$ $[-1,0] \backslash D$ and $y_{2} \in[-1,0] \backslash E$, verifying that

$$
f_{1}\left(x_{1}\right) f_{2}\left(y_{2}\right)=f\left(x_{1}, y_{2}\right)=1=f\left(x_{2}, y_{1}\right)=f_{1}\left(x_{2}\right) f_{2}\left(y_{1}\right) .
$$

Therefore, $f_{1}\left(x_{1}\right) \neq 0 \neq f_{2}\left(y_{1}\right)$, and we have a contradiction because

$$
f_{1}\left(x_{1}\right) f_{2}\left(y_{1}\right)=f\left(x_{1}, y_{1}\right)=0 \text {. }
$$

\section{References}

[1] D. R. Brilleriger, 'Bounded polymeasures and associated translation commutative operators', Proc. Amer. Math. Soc. 18 (1967), 487-491.

[2] I. Dobrakov, 'On integration in Banach spaces VIII (polymeasures)', Czechoslovak Math. J. 37 (1987), 487-506.

[3] _ . 'On integration in Banach spaces IX (integration with respect to polymeasures)', Czechoslovak Math. J. 38 (1988), 589-601.

[4] F. J. Fernández y Fernández-Arroyo, M. L. López García and M. V. Martín del Ama, 'Una integración general en espacios localmente convexos', Rev. Roumaine Math. Pures Appl. 40 (1995), 593-597.

[5] B. Jefferies, 'Radon polymeasures', Bull. Austral. Math. Soc. 32 (1985), 207-215.

[6] B. Jefferies and W. J. Ricker, 'Integration with respect to vector valued Radon polymeasures', J. Austral. Math. Soc. (Series A) 56 (1994), 17-40.

[7] I. Kluvánek, 'Remark on bimeasures', Proc. Amer. Math. Soc. 81 (1981), 233-239.

[8] —_ 'Vector-valued polymeasures and perturbations of semigroups of operators', in: Proc. Miniconference on partial differential equations (Canberra, 1981) (Proc. Centre Math. Anal. Austral. Nat. Univ., 1, Canberra, 1982) pp. 118-123.

[9] H. D. Maynard, 'A Radon-Nikodým theorem for operator valued measures', Trans. Amer. Math. Soc. 173 (1972), 449-463.

[10] K. Ylinen, 'On vector bimeasures', Ann. Mat. Pura Appl. 117 (1978), 115-138. 
Departamento de Matemáticas Fundamentales

Facultad de Ciencias, UNED

Senda del Rey s/n

28040 Madrid

Spain

e-mail: ffernan@mat.uned.es, pjimenez@mat.uned.es, tulecia@mat.uned.es 